\title{
RESET
}

Recherches en sciences sociales sur Internet

$8 \mid 2019$

Genre \& Internet. Sous les imaginaires, les usages ordinaires

\section{La valeur des plaisirs pornographiques en ligne}

Masculinités, sexualités et hiérarchies culturelles

The value of online pornographic pleasures: masculinities, sexualities and

cultural hierarchies

\section{Florian Vörös}

\section{(2) OpenEdition}

1 Journals

\section{Édition électronique}

URL : http://journals.openedition.org/reset/1315

DOI : $10.4000 /$ reset.1315

ISSN : 2264-6221

Éditeur

Association Recherches en sciences sociales sur Internet

Référence électronique

Florian Vörös, "La valeur des plaisirs pornographiques en ligne », RESET [En ligne], 8 | 2019, mis en ligne le 01 juin 2019, consulté le 14 novembre 2019. URL : http://journals.openedition.org/reset/1315 DOI : 10.4000/reset.1315

Ce document a été généré automatiquement le 14 novembre 2019.

(c) Association Recherches en sciences sociales sur Internet 


\title{
La valeur des plaisirs pornographiques en ligne
}

\author{
Masculinités, sexualités et hiérarchies culturelles \\ The value of online pornographic pleasures: masculinities, sexualities and \\ cultural hierarchies
}

Florian Vörös

1 La consommation de pornographie est une pratique largement répandue, inscrite dans les routines de la vie quotidienne d'une grande minorité, voire de la majorité de la population ${ }^{1}$. Dans l'enquête Contexte de la sexualité en France (Bajos, Bozon, $2008: 280$ ), trois femmes sur quatre et neuf hommes sur dix déclarent avoir déjà vu un film pornographique au cours de leur vie. Une femme sur cinq et un homme sur deux déclarent avoir visionné « souvent ou parfois » de la pornographie au cours des douze derniers mois ${ }^{2}$. Si la pornographie prend de multiples formes numériques - des fichiers de films circulant via des sites de pair-à-pair, aux photos amateurs explicites échangées sur des applications de drague, en passant par les sites de petites histoires érotiques la vidéo gonzo ${ }^{3}$ gratuitement accessible via une plateforme de visionnage en streaming constitue aujourd'hui la forme pornographique la plus visionnée (Smith, Barker \& Attwood, 2015). Emblématique du porno des années 2000-2010, cette forme médiatique est aussi la plus décriée, tantôt en raison des modèles économiques ${ }^{4}$ et des idéologies de genre ${ }^{5}$ qui la sous-tendent, tantôt en raison de sa supposée « vulgarité » culturelle ou " perversité » sexuelle ${ }^{6}$. Cependant, internet est aussi le vecteur de discours - émanant de passionnées, de journalistes spécialisées, ainsi que de studios et de plateformes se présentant comme "alternatives", "éthiques» ou "de qualité " (Le Blanc Élie, Lavigne, Maiorano, 2017) - qui insistent sur la pluralité des pornographies et qui reconnaissent une valeur culturelle, sinon à ce genre médiatique dans son ensemble, du moins à certaines de ses réalisations. Partant du constat de la coexistence de logiques de revalorisation et de dévalorisation, ainsi que de l'hypothèse selon laquelle les discours publics ne nous informent qu'indirectement sur la valeur prise par une pratique insérée dans les routines de la vie quotidienne, cet article explore la pluralité 
des modes de dé/valorisation de la pornographie au sein d'un public d'hommes adultes (voir l'encadré méthodologique).

\section{Encadré méthodologique}

De 2008 à 2012, j'ai cherché à m'entretenir avec des hommes usagers de pornographie ayant diverses expériences du genre, de la sexualité et de la classe sociale. Afin de ne pas limiter mon enquête à un public de passionnés, j'ai combiné trois types de recrutement. Une première annonce interpellait des « spectateurs de porno ", qu'ils soient « fans, passionnés ou simples consommateurs occasionnels ». Elle a été diffusée sur deux forums internet de fans de pornographie (hétérosexuelle), ainsi que sur deux sites de rencontre gays. Deux autres annonces, l'une ciblant des hommes homo et bisexuels, l'autre ciblant des hommes hétérosexuels, ne mentionnaient la pornographie que dans une liste plus large de représentations audiovisuelles de la sexualité. À travers ces trois modes de recrutement, je me suis entretenu avec quatorze hommes gays, treize hommes hétérosexuels, trois hommes bisexuels, deux lesbiennes, une femme bisexuelle et un trans' Female-to-Male (FtM) ${ }^{7}$. Au sein de ce public, les cadres d'entreprise, les cadres de la fonction publique et les professions intellectuelles et supérieures sont surreprésentés ${ }^{8}$. Tous visionnent de la pornographie - de manière quotidienne, hebdomadaire ou plus occasionnelle - le plus souvent sur des sites de streaming, via un ordinateur, lorsqu'ils se retrouvent seuls à leur domicile. Avec moins de fréquence, ils en visionnent aussi dans d'autres formats (DVD, vidéo à la demande, fichiers téléchargés de pair-à-pair), sur d'autres appareils (télévision, smartphone), dans d'autres lieux (sex-shops, sex-clubs, au bureau, dans les transports en commun) et dans d'autres configurations interactionnelles (en couple, avec un partenaire occasionnel, en groupe).

Les entretiens - conduits en face-à-face, d'une durée d'une à six heures et parfois répétés - m'ont permis de recueillir des informations relatives aux pratiques spectatorielles (le guide d'entretien s'ouvre sur des questions sur « ton histoire de spectateur », «tes goûts » et " tes habitudes ») ainsi qu'aux modes de vie (« toi et ton entourage ») et aux visions du monde (l'entretien se termine sur « ce que tu penses du porno »). Ces entretiens - conduits tantôt à mon domicile tantôt à celui des participants à l'enquête - m'ont également permis d'observer des présentations de soi, par la manière de se tenir et de se raconter face à un enquêteur homme, qui se présente quant à lui comme doctorant en sociologie et passionné de pornographie ${ }^{9}$. Après les entretiens, la retranscription de la parole des participants s'est accompagnée de la reconstitution de leurs parcours de navigation en ligne, à partir des données recueillies relatives aux sites web fréquentés et aux mots-clés utilisés dans les moteurs de recherche.

2 Au-delà de la diversité des usages - lutter contre l'ennui et le désœuvrement, s'évader, pallier une sexualité jugée insuffisante, renforcer une relation amoureuse, s'informer, interroger ses sensations et ses fantasmes, rencontrer des partenaires sexuels, etc. (Smith, Barker, Attwood, 2015) - le goût pour la pornographie s'énonce avant tout comme un attachement à un état corporel et à une intensité d'expérience. Un «bon » porno, c'est d'abord un porno qui " excite ", « émoustille ", « titille ", « émeut », " fait bander», «fait bien triquer» ou «fait mouiller la culotte», pour reprendre les 
expressions les plus utilisées par les participants à l'enquête. Les spectateurs rejoignent en cela les journalistes spécialisés, dans leur habitude d'évaluer les films et vidéos pornos à l'aune de leur potentiel d'excitation sexuelle, laquelle est souvent représentée de manière masculine, sous la forme de l'érection (Williams 1991; Trachman, 2013; Damian-Gaillard, 2014). Les plaisirs pornographiques sont tout autant tactiles qu'audiovisuels (Paasonen, 2011): il ne s'agit pas seulement de voir des images et d'écouter des sons mais aussi de manipuler un ordinateur et de stimuler des zones érogènes, éventuellement avec du lubrifiant et des sex-toys. L'approche spinozienne des affects en jeu dans les pratiques de navigation en ligne (Pailler, Vörös, 2017), en ce qu'elle s'intéresse à la fois aux modifications du corps (affectio) et aux idées relatives à ces modifications (affectus) (Spinoza, 2005 : 197), permet d'appréhender le plaisir à la fois comme un événement sensible (Deleuze, 1981; Hennion, 2007) et comme une structure de significations socialement déterminée et hiérarchisée (Bourdieu, 1979; Lahire, 2004a).

Illustration $1:$ Quatre modalités idéales-typiques

\begin{tabular}{|l|l|l|}
\hline & Investissement positif & Investissement négatif \\
\hline Investissement fort & Passion & Contrôle \\
\hline Investissement faible & Satisfaction & Distinction \\
\hline
\end{tabular}

3 Le tableau 1 distingue quatre modalités idéal-typiques d'organisation des plaisirs pornographiques: la "satisfaction » est un investissement faible et positif dans la pratique, la « distinction » est un investissement faible et négatif, le « contrôle » est un investissement fort et négatif, et la "passion» est enfin un investissement fort et positif. Cette typologie a été élaborée en croisant les récits de pratiques des participants à l'enquête, mon expérience de reconstitution de leurs parcours de navigation en ligne, ainsi que la théorie des «sensibilités culturelles" élaborée par Lawrence Grossberg - initialement pour rendre compte de la spécificité de la "sensibilité fan " par rapport aux autres formes d'organisation des plaisirs issus du divertissement et des industries culturelles. Dans la tradition des Cultural Studies, il s'agit de "prendre au sérieux les différences " - en l'occurrence liées au genre, à la sexualité et à la classe sociale - « entre les différentes fractions des publics de la culture populaire » (Grossberg, 1992 : 53). Deux précautions préliminaires sont ici nécessaires. D'abord, ces idéaux-types, en tant que produit d'une opération de réduction logique, ne représentent pas des spectateurs individuels, dont le rapport à la pornographie est toujours complexe et ambivalent, mais des modalités d'action de soi sur soi (Foucault, 1984). Ainsi, différents modes peuvent coexister au sein d'une même expérience individuelle. Ensuite, ces modalités d'action n'appartiennent pas a priori à un groupe social plutôt qu'à un autre. Elles sont en revanche des techniques de soi qui participent de la production de subjectivités genrées.

4 Dans les développements qui suivent, il s'agit de comprendre comment ces processus de dé/valorisation de la pornographie peuvent participer de processus de construction de la masculinité, c'est-à-dire de définition de soi en tant qu'homme, en rapport aux femmes et à d'autres hommes (Connell, 2014). Dans un contexte de controverses 
récurrentes relatives aux rapports de domination reproduits par les représentations pornographiques et leurs conditions de production (Cornell, 2000), nous prêterons une attention particulière aux réactions (curiosité, désintérêt, rejet, etc.) que chacune de ces modalités encourage, parmi les publics masculins, vis-à-vis des démarches de politisation féministe des plaisirs pornographiques.

\section{Se satisfaire de la pornographie}

5 Le mode de la satisfaction envisage les plaisirs pornographiques comme limités dans le temps et dans l'espace. Les objets pornographiques sont ici envisagés comme des instruments et les pratiques de visionnage remplissent une fonction simple : il s'agit d'éveiller ou d'assouvir des "envies» et des «besoins", le plus souvent seul et éventuellement à plusieurs. Ce mode requiert des savoir-faire numériques et sexuels de production du plaisir qui permettent de trouver, au sein des différents univers pornographiques en ligne, les mots, les images, les sons et les scénarios les plus à même de parvenir à une satisfaction sexuelle rapide. Les effets des pratiques pornographiques sont alors comparés à ceux d'une prise de drogue qui désinhibe ("se lâcher »), d'un échauffement qui prépare ("se chauffer») ou de la dégustation d'un condiment en accompagnement d'un plat (« pimenter »).

6 Le mode de la satisfaction investit le plaisir tactile du processus de navigation - le clic, le passage en revue, le balayage d'écran, l'avance rapide - davantage que les singularités de chacune des vidéos pornographiques. Ces dernières, sur lesquelles on tombe en navigant de tags en tags (en cliquant sur les mots-clés qui indexent les vidéos) sont ici interchangeables, dans la mesure où elles participent de ce processus de production de plaisir. À l'instar du feuilletage de magazines (Nixon, 1992) ou du zapping télévisé, le plaisir dérive de l'impression d'abondance, du défilement des images et de la manipulation des objets. Le passage de la rareté à l'abondance est un thème récurrent parmi les spectateurs qui ont, comme Michel (43 ans, bisexuel, cadre commercial) découvert la pornographie avant les années 2000 et la généralisation de l'internet haut débit et du développement des sites de visionnage gratuit en streaming: " Tout l'intérêt de cette pléthore d'offre sur internet, c'est que tu peux zapper très vite et c'est sans cesse renouvelé ». Ce sentiment de nouveauté peut toutefois coexister avec l'impression de « revoir toujours la même chose ». Le plaisir consumériste peut alors se transformer en une lassitude voire en un dégoût vis-à-vis d'une «hyperconsommation" à travers laquelle " on finit par consommer beaucoup sans vraiment regarder» (idem).

7 Le mode de la satisfaction peut reprendre certaines des formes conventionnelles de l'expression de la sexualité masculine comme une force naturelle qui jaillirait de l'intérieur et qu'il s'agirait d'extérioriser ou de juguler. Les pratiques pornographiques masculines y sont souvent racontées à travers des métaphores mécaniques qui convoquent le champ lexical du relâchement et de l'extériorisation de liquide et de gaz: "faire la vidange" "soulager la cocotte-minute", "se vider les couilles » ou "décompresser ».La métaphore du médicament est également courante, celui-ci pouvant être un " complément " à des relations insatisfaisantes comme un "substitut " à l'absence de relation ${ }^{10}$. Moins qu'une pratique aphrodisiaque, le recours à la pornographie devient dans ce second cas une manière de prendre soin de soi : "un petit plaisir solitaire en période de disette " (Marc, 47 ans, bisexuel, célibataire, 
ingénieur) voire un «palliatif à des souffrances » (Frédéric, 28 ans, gay, célibataire, sans emploi). Ce registre du soin n'est formulé qu'à demi-mots lorsqu'il entre en tension avec une construction plus conventionnelle de la masculinité: si Julien (28 ans, hétérosexuel, chef de produit touristique, célibataire) évoque "des moments de passage à vide où [il] en regardai[t] beaucoup ", il ne s'attarde pas sur ces difficultés et met en avant sa capacité à l'autonomie et son invulnérabilité émotionnelle.

Les « bonnes » vidéos pornographiques sont ici celles dont l'esthétique est " plaisante », "agréable » et "confortable». Sélectionner une belle vidéo pornographique haute définition, avec de "belles images », «bien cadrées » et des " couleurs intenses » est pour Antoine ( 35 ans, gay, magasinier) l'assurance de passer un bon moment le soir à son domicile avec son compagnon. Ces qualificatifs désignent à la fois les objets pornographiques et les ambiances sexuelles qu'ils permettent d'activer. En dehors de ce périmètre restreint de la sexualité récréative, la pornographie perd toutefois sa valeur et il n'y alors plus vraiment d'intérêt à en discuter. Par exemple, Gaëtan (35 ans, gay, commerçant) visionne tous les jours des vidéos pornographiques amateurs sur des porn tubes mais ce n'est pas une pratique qui fait l'objet de conversations car elle « est vraiment réservée à [s]on plaisir solitaire de trois minutes par jour ». Aussi, envisager la pornographie avant tout par rapport à ses "effets", à sa fonction somatique, induit une disjonction entre les goûts pornographiques et les pratiques spectatorielles plus « intellos ». Tom ( 29 ans, lesbienne, petits boulots) ne situe " pas au même niveau » le cinéma et le porno car ces deux types d'objets ne lui "servent pas à la même chose »: " les pornos, qu'ils soient gays ou hétéros, [elle] n'en a jamais vus en entier » et elle " ne fai[t] pas trop gaffe à ce qu'il y a autour » dans la mesure où elle "les regarde avant tout pour se branler ». La valorisation du plaisir pornographique est ici limitée dans l'espace et dans le temps : elle opère dans le cadre d'une sexualité récréative, le plus souvent domestique et individuelle.

Le mode de la satisfaction dessine ainsi un rapport fonctionnel et utilitariste au plaisir pornographique. Au sein du public enquêté, ce sont les hommes dont le statut social dépend moins du capital culturel que du capital économique (commerçants, magasiniers, attachés commerciaux, cadres commerciaux, ingénieurs) qui l'énoncent avec le plus de clarté et de facilité. Cette relation "sans prise de tête " aux images peut s'associer au rejet de toute forme d'intellectualisation de réflexion critique sur la pratique. Par exemple, lorsque je demande en fin d'entretien à Pascal ce qu'il pense des critiques féministes de la pornographie hétérosexuelle, il me répond : «Pour moi, ça m'excite, ça me fait me branler. Point. " (Pascal, 46 ans, gay, attaché commercial). Envisager la pornographie par rapport à son seul potentiel d'excitation sexuelle peut ainsi conduire au désintérêt vis-à-vis des enjeux politiques qui la traversent.

\section{Se distinguer de la pornographie}

Tandis que le mode de la satisfaction se contente de disjoindre le divertissement pornographique des pratiques spectatorielles jugées plus intellectuelles, le mode de la distinction valorise l'intellectuel au détriment du corporel. Depuis son invention moderne au XIXe siècle, la pornographie est considérée par les élites culturelles comme le « nadir de la culture » en ce qu'elle « offense les convenances corporelles et sexuelles qui soutiennent les distinctions de classe : les bonnes manières, la pudeur, la dignité, l'effacement des instincts corporels et leur sublimation en un comportement policé » 
(Kipnis, $2015: 39$ ). Si la pornographie est bien une affaire de légitimité culturelle et de rapports de classe, une enquête qualitative en réception permet de nuancer cette analyse et de mettre au jour des dissonances intra-individuelles (Lahire, 2004a) générées par ce mode légitimiste d'appropriation de la pornographie, ici surtout porté par des hommes dont la position sociale dépend fortement du capital culturel (libraires, artistes plasticiens, enseignants-chercheurs, chargés de communication, cadres administratifs).

11 La vidéo pornographique en ligne est présentée comme laissant peu de place à l'imagination, à la différence de l'érotisme littéraire. Trop «commerciale » et «standardisée », elle est jugée insuffisamment «artistique» et "créative». La comparaison alimentaire est ici celle de la malbouffe et du fast-food, des produits gras, chimiques et indigestes de l'industrie agro-alimentaire; et cela peut aller jusqu'à comparer les acteurs et les actrices à de la "viande » dépourvue d'intelligence. La dévalorisation de la pornographie articule alors des logiques de classe et de genre: les mises en scène hyperboliques de la virilité (gros muscles, gros pénis) et de la féminité (gros seins, faux ongles, maquillage appuyé) sont décodées comme "grossières » et "vulgaires", assimilées au "mauvais goût » des classes populaires et des "nouveaux riches ». Élevé dans une famille "très stricte, très scolastique, très académique » de la côte Est des États-Unis, Andrew (28 ans, gay, chargé de communication) affiche par exemple un dégoût à la fois gay et bourgeois pour la «très basse qualité » de la pornographie hétéronormée nord-américaine, qu'il juge "kitsch » et " caricaturale ", et qu'il oppose à l'esthétique «sobre » et "minimaliste» de revues érotiques gays européennes telles que Butt ou Fantastic Man.

La distinction vis-à-vis des « autres » que seraient les « consommateurs », les « fans » et les «accros » de la vidéo pornographique en ligne vise aussi une part illégitime de soimême. Ainsi, Michel (43 ans, bisexuel, cadre administratif) explique que sa " consommation de pornographie à haute dose " lui pose " un problème entre moi et moi ». D'un côté, il « aime beaucoup, honnêtement, toutes les formes pornographiques, qu'elles soient hétéros, bisexuelles ou gays ». De l'autre, cette pratique est problématique dans la mesure où il a l'impression de consacrer à la pornographie plus de temps qu'elle ne mérite. Michel met ainsi en scène une lutte intra-individuelle (Lahire, 2004a) entre deux dynamiques de subjectivation concurrentes : d'un côté, l'état de plaisir et de bien-être que lui procure la navigation pornographique, de l'autre, l'adhésion à une norme de bon comportement culturel qui l'amène à penser qu'il « vaut mieux » que la pornographie. En lieu de la reconnaissance de ce dilemme, le mode de la distinction conduit souvent à une relativisation de l'importance de l'attachement à la pornographie. D'autres participants à l'enquête présentent alors le recours occasionnel à la pornographie comme une anecdote insignifiante. Le statut de « consommateur de porno " - terme négativement connoté par les participants à l'enquête - est alors relativisé pour mettre en avant d'autres pratiques de lecture et de visionnage perçues comme plus valorisantes. Bernard (54 ans, médecin, gay) entrecoupe par exemple le récit de ses pratiques pornographiques de digressions sur d'autres activités qui seraient plus signifiantes au quotidien, telles que la lecture du journal Le Monde. Non seulement il "ne regarde pas que ça " mais il consacre le moins de temps possible au divertissement audiovisuel en général. Dans le cadre de la relation d'enquête, le mode de la distinction opère comme un refus de se voir coller l'étiquette péjorative de « consommateur de porno ». Lorsque j'amène la question de la pornographie dans le 
cadre de notre entretien sur «les représentations de l'homosexualité masculine à l'écran", cela suscite une réaction défensive de la part de Philippe (employé de librairie, gay, 36 ans, en couple) qui me recadre tout de suite afin de préciser qu'il n'est pas un "gros consommateur " et encore moins un "collectionneur de films pornos, loin de là ». De la même manière, lorsque je lance Nicolas (29 ans, hétérosexuel, ingénieur de la vision en cinéma) sur ses goûts et ses habitudes en matière « d'amour et de sexualité à l'écran ", tout en lui précisant que je suis particulièrement intéressé par son rapport à la pornographie, il me répond que le porno «n'a pas d'importance dans [sa] vie » et que « ça ne définit pas qui [il est] ».

$13 \mathrm{Au}$ quotidien, le statut de " collectionneur » est évité à travers la dissimulation de ses traces d'usage, par exemple en effaçant régulièrement l'historique de son navigateur web. Le spectre du « fan » - autre terme péjoratif selon les participants à l'enquête - est pourchassé à travers l'oubli des noms des acteurs, actrices, réalisateurs, réalisatrices. Par exemple lorsque j'invite Marc (47 ans, bisexuel, ingénieur) à me donner des informations plus précises sur ses goûts pornographiques (il m'expliquera par la suite qu'il a «honte» d'aimer les vidéos du studio Citébeur), il m'oppose qu'il n'est « pas certain de pouvoir dire quelle marque réussit toujours ça ou quelle marque réussit moins bien ", comme si je l'interrogeais sur ses pratiques d'achat en supermarché. Andrew (28 ans, chargé de communication) s'excuse quant à lui de se rappeler de " choses aussi frivoles et stupides " que le nom du réalisateur et acteur porno Michael Lucas.

14 Tandis que des acteurs et des actrices de l'industrie pornographique se mobilisent autour du slogan «\#PayForYourPorn" ${ }^{11}$ " (littéralement " paye pour ton porno!»), le visionnage de contenus gratuits peut être un moyen, pour les spectateurs, de ne pas se voir accoler l'étiquette péjorative du «consommateur de porno». Ce comportement s'inscrit à la fois dans des habitudes d'accès gratuit aux contenus numériques et dans un refus plus spécifique de se laisser identifier, à travers l'acte d'achat, par un objet qui pourrait venir "salir» (Frédéric, 28 ans, gay, architecte) une identité culturelle respectable. En effet, « l'acte de payer n'a pas seulement une signification économique "; cet acte implique également d'accepter de s'identifier et d'être identifié par l'objet acheté. Payer pour un film pornographique devient alors "payer de sa personne " (Lahire, 2004b : 103) au sens d'accepter que cet objet résume, sinon toute, du moins une partie de soi-même, de ses préférences, de ses goûts et de son mode de vie.

15 Le mode de la distinction envisage la pornographie comme une souillure qui viendrait entacher une image légitime que l'on se fait de soi-même et que l'on veut communiquer à autrui. C'est ainsi que ce mode décourage la perception et l'expression de soi en tant que membre du public et la réflexion critique sur les implications politiques de ses pratiques pornographiques.

\section{Contrôler son « addiction » à la pornographie}

16 Ce troisième mode d'organisation du plaisir consiste en un effort pour se ressaisir et limiter l'emprise de la pornographie sur son mode de vie. À la différence du mode de la distinction, il s'appuie sur une grille de lecture médicale. La perception de soi en tant qu'« accro » à la pornographie ouvre à la mise en place de résolutions personnelles visant la restriction du périmètre de la pratique, voire son arrêt total. 
17 Si la pornographie est décrite comme une bonne drogue lorsque son effet est plaisant et que son emprise est circonscrite (mode de la satisfaction), elle devient une mauvaise drogue lorsqu'elle est associée à la notion de perte de contrôle. Passionné de pornographie depuis son adolescence, Pierre (44 ans, hétérosexuel, célibataire, cuisinier) devient " vraiment addict » à la trentaine suite au divorce avec son épouse et son emménagement dans un studio où il se retrouve pour la première fois connecté à l'internet haut débit. Le soir en rentrant du travail, il lui arrivait alors de «naviguer jusqu'à quatre ou cinq heures du matin, jusqu'à [s']abimer les yeux » et, lors de ses jours de repos, il pouvait passer «jusqu'à quinze heures» devant son ordinateur. La pornographie devient ainsi une activité qui " prend beaucoup trop de temps » et autour de laquelle il finit par se «renfermer ». Il distingue les long-métrages de qualité qu'il visionnait avant en DVD et les vidéos en ligne de style gonzo qu'il télécharge de pair-àpair, qui sont selon lui «toujours la même chose : une nana, un canapé et bam bam », mais vis-à-vis desquelles il entretient « une fascination malsaine ». Il raconte le dégoût envers ces images et envers son propre comportement à travers la métaphore des troubles du comportement alimentaire: "Je bouffais mon sandwich ou mes brocolis comme un geek devant son écran [...] Tu te gaves [...] Tu consommes, et vu que c'est gratos, tu consommes encore plus ». Face à ce constat, Pierre se « ressaisit ». Il en vient à se considérer comme "addict » et à envisager la pornographie en ligne comme une «drogue ». Considérant qu'il n'est plus en mesure de gérer son usage d'internet de manière autonome, il décide de résilier son abonnement et de faire couper sa ligne téléphonique. Désormais, Pierre consulte son courrier électronique depuis un cyber café, où il se sent surveillé et parvient à se concentrer exclusivement sur les activités numériques non-sexuelles. Cette reprise en main est racontée à travers un récit du passage de « l'addiction » à la « libération » dont les codes ont été posés par un discours sexologique fortement médiatisé (Vörös, 2009) :

Je n'ai plus envie d'être prisonnier de ce monde virtuel. Je me suis dit qu'il fallait que je fasse un vrai sevrage. Comme un drogué. Parce que j'étais un drogué ! Il fallait que je coupe. [...] C'est comme si un mec arrête de fumer, mais reste dans un environnement fumeur [sourit], ça ne tient pas. Je me suis dit qu'il y avait quelque chose de pas logique dans ma volonté d'arrêter. C'est comme si j'ai du shit ou de la beuh chez moi : je suis incapable de ne fumer que le week-end. J'ai du bon matos, je fume ! Avoir internet chez soi, c'est pareil. Tu rentres chez toi, tu allumes ton écran d'ordinateur à la con, et tu ne peux pas t'en empêcher. Tu ne peux pas t'arrêter, parce que c'est devant toi, simple et gratuit ! [Pierre, 44 ans, hétérosexuel, célibataire, cuisinier].

18 Le récit de Mathias (41 ans, hétérosexuel, projectionniste, célibataire) converge en de nombreux points avec celui de Pierre. Pour lui, qui vit également seul en studio, l'équipement en internet haut débit à domicile et la découverte des sites de visionnage gratuit en streaming ont aussi donné lieu à une autosexualité domestique difficile à contrôler. Face à l'abondance de vidéos sur les porn tubes, Mathias cherche celles qui lui procurent les sensations les plus fortes possibles. Il perd progressivement le contrôle sur cette quête d'intensité et l'excitation se teinte de lassitude et de dégoût.

Il y a des images choquantes, et je vais vers les images choquantes, vers une espèce d'obscénité, qui me choque. Sauf qu'avec le temps, tu vois toujours les mêmes choses et tu te lasses, tu en as marre, tu satures [Mathias, 41 ans, hétérosexuel, projectionniste, célibataire].

19 Ne supportant plus de passer « quatre à cinq heures par jour, parfois la nuit entière » à naviguer sur des sites pornographiques en quête de chocs sensoriels, Mathias engage 
alors un travail réflexif, à travers lequel des sensations négatives (le dégoût et la lassitude) sont réévaluées en pathologie à soigner (l'addiction) : "Ça s'est organisé autour d'un dégoût. Un matin, je me suis dit, je vais taper "accro" et "pornographie" sur mon moteur de recherche ". La "guérison" passe chez Mathias par un arrêt temporaire de la pratique, qu'il qualifie successivement de "diète ", de "jeûne ", de "cure» et de "sevrage». Au moment de l'entretien, Mathias vient toutefois de se séparer de sa compagne et de faire une "rechute». L'élément déclencheur a été la consultation en librairie d'une bande dessinée sadomasochiste mettant en scène "la soumission de la fille » et « la jouissance sadique des mecs ».

Le mode de l'addiction s'exprime différemment selon le statut conjugal et la culture sexuelle. Les hommes hétérosexuels monogames ou célibataires (comme Pierre et Mathias) parlent surtout d'une dépendance à l'autosexualité pornographique. La perte de contrôle est décrite par ces hommes comme un glissement progressif d'une frénésie joyeuse vers le dégoût, l'écœurement et la honte. Aussi, ils décrivent l'addiction comme une course en avant vers des images impliquant des mises en scène de la domination masculine et de la soumission féminine de plus en plus spectaculaires. Les mouvements féministes interrogent depuis les années 1970 les idéologies conservatrices à travers lesquelles « les hommes ne peuvent s'imaginer que dirigeant et contrôlant les femmes" (Mathieu, 2013 : 152). Le mode du contrôle réduit cet enjeu politique - la force de l'interpellation idéologique des imaginaires sexuels hétéronormés (Butler, 2005) - à une simple question de bien-être individuel, coupant court à tout exercice d'introspection sur sa propre implication dans des mécanismes de domination masculine.

21 Le récit hétérosexuel masculin de «l'emprisonnement » à la «libération » prend souvent la forme d'une affirmation hétéronormative de la masculinité. Mathias raconte par exemple sa "sortie de l'addiction» comme un processus de normalisation masculine : alors qu'il ne parvenait plus à éjaculer dans le cadre d'une sexualité à deux, l'arrêt de la masturbation pornographique lui a permis de redevenir un «mâle éjaculateur». Se "libérer» de la dépendance lui permet ainsi de « retrouver » sa force virile dans le domaine de la sexualité. Louis (30 ans, hétérosexuel, ingénieur) perçoit quant à lui plus largement l'ensemble des technologies domestiques comme recelant un risque d'affaissement de la virilité, par contraste avec les activités sportives en plein air. Il en vient à rejeter ensemble la pornographie, la technologie et le féminisme au nom de la défense de la virilité. Louis, comme d'autres, voit la réduction des pratiques de masturbation pornographique comme une manière de reprendre le contrôle sur sa sexualité (Taylor, Jackson, 2018) et son pouvoir sur les femmes (Gourarier, 2017).

Les hommes gays et bisexuels multipartenaires rapportent davantage une dépendance aux échanges via des sites de rencontres et des applications mobile. Par exemple, pour Sébastien (37 ans, gay, en couple, enseignant dans le secondaire), le récit de l'addiction ne prend pas pour objet spécifique le visionnage de contenus pornographiques, mais décrit plus largement un mode de vie multipartenaire orienté vers la recherche du contact corporel.

Je fais beaucoup plus de chat que de porno. C'est-à-dire que, pour moi, le premier plaisir sexuel, ce n'est pas le porno, c'est vraiment la relation avec le mec. C'est avant tout le feeling, l'animalité, l'attraction qu'il peut y avoir [Sébastien, 37 ans, gay, en couple, enseignant dans le secondaire]. 
Sébastien parle d'addiction pour désigner l'état dans lequel il se retrouve lorsqu'il passe plusieurs heures d'affilée à naviguer sur des sites de drague, à la recherche de partenaires sexuels, en reportant à plus tard ses obligations professionnelles.

Ça me fait perdre énormément de temps. Je ne sais pas si le sexe est devenu une drogue, je ne sais pas si je suis un sex addict - je pense pas - mais, en tout cas, je peux perdre beaucoup de temps là-dessus. Je peux passer trois heures, une aprèsmidi entière, sur les chats. Parce que j'ai envie de baiser, et que je trouve pas. Et donc ça fait trois heures de foutues en l'air, alors que j'ai un taf monstre à côté [Sébastien, 37 ans, gay, en couple, enseignant dans le secondaire].

L'addiction est ici évoquée pour décrire une situation où Sébastien peine à cantonner le sexe et la drague, loisirs qui lui procurent du plaisir, dans un espace raisonnable, compatible avec son travail d'enseignant et les multiples projets artistiques dans lesquels il est par ailleurs investi. Sébastien "ne pense pas être malade pour l'instant ", car même si sa sexualité "prend quand même une grande place ", il parvient le plus souvent à en contrôler l'emprise. L'action de soi sur soi qui consiste à "guérir » est ici indissociable de la norme d'une vie professionnelle et culturelle riche et exigeante. Parmi ceux qui participent aux espaces de la sexualité gay multipartenaire, le vocabulaire de la perte et de la reprise de contrôle renvoie ainsi à une tension entre utopie d'un monde tourné autour du plaisir sexuel et un monde fait d'obligations professionnelles.

Les hommes qui s'appliquent le vocable de l'addiction, afin de reprendre le contrôle sur leur rapport à la pornographie n'ont pas un rapport strictement négatif à la pratique. L'appréhension de la pornographie comme une mauvaise drogue (mode de l'addiction) côtoie en effet souvent son appréhension comme une ressource sensorielle et émotionnelle utile (mode de la satisfaction), voire comme une passion à part entière.

\section{Se passionner pour la pornographie}

Le mode de la passion amplifie et prolonge le plaisir pornographique, à travers un enthousiasme qui perdure au-delà de l'espace-temps du visionnage, pour inscrire la pratique dans des réseaux de sociabilité et lui conférer une publicité. Cette revalorisation des plaisirs pornographiques repose sur des régimes de valeur alternatifs (Glévarec, 2005 ; Maigret, 2012) élaborées au sein de subcultures sexuelles.

27 En opposition au mode de la distinction, qui dévalue la pornographie en identifiant ses manques esthétiques, le mode de la passion juge les films et vidéos pornographiques à l'aune de critères de jugement spécifiques, afin d'apprécier la spécificité de l'expérience spectatorielle offerte par ce genre médiatique. Plutôt que d'évaluer les pornographies qu'il apprécie en les comparant à des genres plus légitimes, Wayne (30 ans, FtM, pansexuel, en couple, étudiant en cinéma) insiste sur le fait qu'il «aime vraiment le porno en soi ». Le porno est selon lui le seul genre cinématographique à proposer « une expérimentation avec le corps». "Regarder du porno pour regarder du cul » n'est selon lui pas contradictoire avec la recherche esthétique de «l'étincelle » qui rend ce spectacle "hyper beau». Fabrice ( 44 ans, hétérosexuel, célibataire, technicien son, participe à un forum de passionnés) ne nie pas le fait que, en tant que cinéma de genre, "le gonzo c'est quelque part toujours la même chose", mais cela n'est pour lui pas contradictoire avec le fait qu'il s'agisse d'un « art de faire passer les sensations » :

RESET, 8 | 2019 
Je suis grand fan de cinéma, je vais souvent en voir en salles, et je le regarde aussi pas mal à la télé. Et je ne supporte pas le porno quand il veut ressembler au cinéma traditionnel. Moi, je considère que le porno, c'est vraiment un cinéma de genre, comme le gore, comme le western. Je pense que c'est un cinéma entre guillemets un peu extrême. Un peu comme pour certains genres de musique, qui sont assez extrêmes. C'est un cinéma de genre qui se suffit à lui-même [Fabrice, 44 ans, hétérosexuel, célibataire, technicien son, participe à un forum de passionnés].

Le mode de la passion établit des ponts entre la pornographie et un répertoire plus large de pratiques culturelles. Là où la cinéphilie traditionnelle marginalise la pornographie, le mode de la passion en fait un point nodal. La pornographie peut par exemple être envisagée dans le cadre d'un intérêt plus large pour les « genres filmiques corporels » (Williams, 1991 ; Dyer, 2015) qui visent à susciter dans le corps du public les états corporels représentés à l'écran. Wayne fait ainsi une analogie entre sa passion pour la vidéo porno et le film d'horreur, deux « cinémas de genre » qui sont «basés sur la sensation ». Il aime particulièrement le côté «trash» et "gore » du porno zombie, qui hybride ces deux genres. Aussi, sa passion pour la musique des pornos états-uniens des années 1970 et des pornos de « gangsters » et de « lascars » des années 2000 renvoie à son goût pour les cultures hip-hop et disco. Bertrand (42 ans, hétérosexuel, célibataire, petits boulots, participe à un forum, tient un blog) pratique quant à lui ce qu'il appelle le «psychédélik porn», qui hybride porno et culture hippie. Concrètement, cela consiste en de la peinture abstraite sur des collages de photos de magazines pornos et en des remix de vidéos pornos associés à de la musique de transe psychédélique. Bertrand met en avant une sensibilité kitsch, qui valorise le second degré et le sens de l'autodérision présent dans certaines productions pornographiques :

Les miches de la boulangère ${ }^{12},[. .$.$] c'est l'histoire d'une p'tite nana qui travaille chez$ le boulanger comme stagiaire. Il la fait s'habiller avec des bas et des portejarretelles. Là, on est vraiment dans le porno classique [sourit], que tout le monde critique, mais moi j'aime bien ce côté bancal, mal joué, ces scènes absurdes. [...] J'aime bien aussi La Belle des Vosges ${ }^{13}$, qui est vraiment un pur navet nanar à petit budget. J'aime bien ce film, parce que pour moi le côté ridicule ça fait partie du porno [Bertrand, 42 ans, hétérosexuel, célibataire, petits boulots, participe à un forum, tient un blog].

Le rire moqueur sur la pornographie côtoie ici le rire enthousiaste avec la pornographie. Michel (43 ans, bisexuel, célibataire, cadre administratif) aime le côté «absolument délirant» des pornos péplums qui se déroulent dans une "fausse Rome en cartonpâte » et qui assument une vision "stéréotypée ». Wayne (30 ans, FtM, pansexuel, en couple, étudiant en cinéma) aime quant à lui les acteurs de porno avec lesquels il " arrive à [se] marrer et à [se] branler en même temps ". De la même manière, les critiques de film pornos que Pierre (44 ans, hétérosexuel, célibataire, cuisinier, membre d'un forum) publiait via le Minitel dans les années 1990 se distinguaient par leur ton «fendard ». Alors que le rire et l'excitation sont souvent présentés comme deux affects contraires, le mode de la passion les réunit ainsi en un même enthousiasme. La légitimité des plaisirs pornographiques reste cependant fragile dans la mesure où le rire peut également venir signifier la gêne voire la honte.

Les subcultures sexuelles minoritaires jouent historiquement un rôle moteur dans les processus de légitimation de la pornographie (Rubin, 2011b). Pour Wayne, le développement d'une connaissance du porno gay à partir de la vingtaine a participé d'un mouvement plus large de socialisation aux mondes gays et queer à travers la fréquentation de groupes militants, de librairies, de ciné-clubs et de festivals de 
cinéma. L'investissement dans une subculture sexuelle minoritaire peut ainsi venir rapprocher le porno d'objets culturels plus visibles, comme la musique pop, ou plus légitimes, comme le cinéma d'auteur ou expérimental. Aussi, pour les spectateurs fortement investis dans une sociabilité sexuelle gay, l'échange de photos et de vidéos amateurs (éventuellement érotico-pornographiques) via des sites web et des applications mobiles est souvent coextensif de la fréquentation d'espaces physiques (bars, discothèques, concerts, etc.) où l'on recroise les hommes vus sur internet. Pour Gaëtan (35 ans, commerçant, gay), adepte du site XTube, la plus-value de la vidéo porno amateur par rapport à la pornographie professionnelle est que, lorsque la vidéo est rattachée au profil personnel d'un des protagonistes, il a la possibilité de contacter ce dernier avec l'espoir de rejouer cette scène. Sa présence en ligne lui permet d'approcher de nouveaux partenaires sexuels et d'entretenir les relations existantes avec des partenaires réguliers. La mise à jour régulière des textes, des photographies et des vidéos qui composent son profil personnel XTube vise à soigner son image publique - selon une logique de "microcélébrité » (Marwick, boyd, 2011) - auprès d'hommes qu'il peut également croiser à la gym ou dans les rues du Marais. Cet usage relève du «modèle du réseau sexuel» selon lequel «l'activité sexuelle apparait aux individus comme une composante ordinaire de leur sociabilité, génératrice de capital social, mais également créatrice de liens d'interdépendance » (Bozon, $2001: 16$ ).

31 Contre l'adage selon lequel "quand on en a vu un on les a tous vu », la passion repose sur l'élaboration d'échelles de valeur spécifiques à la pornographie, qui permettent de distinguer et de hiérarchiser les productions appartenant à ce genre médiatique. La métaphore alimentaire est ici celle de la gastronomie. Fabrice (44 ans, hétérosexuel, célibataire, technicien son, participe à un forum de passionnés) souligne l'importance pour le "gourmet» de bien connaître les «ingrédients » et les «recettes » qui permettent d'obtenir la meilleure vidéo porno. Les passionnés tendent à insister davantage sur l'importance de la réalisation que sur celle des corps et des pratiques représentées. Faisant le constat que "tout le monde ne sait pas tourner un film X ", Pierre (44 ans, hétérosexuel, célibataire, cuisinier, participe à un forum) privilégie les films tournés par des réalisateurs qui ont à la fois fait des études de cinéma et qui ont une expérience conséquente dans le X. Les passionnés disent aussi se repérer davantage par des noms propres que par des mots-clés. Wayne ( 30 ans, FtM, pansexuel, étudiant en cinéma) n'est pas du genre «à taper dans un moteur de recherche une pratique sexuelle en particulier ", car "si elle est mal filmée, ça n'aura pas d'intérêt ». La recherche de qualité pousse les passionnés à l'achat, malgré l'accès facile à des contenus gratuits. Gérard (62 ans, hétérosexuel, célibataire, retraité, participe à un forum) n'utilise par exemple internet que pour s'informer sur les nouveautés, lire les opinions des forumeurs et des bloggeurs et acheter des DVD qu'il visionne ensuite sur grand écran pour en apprécier la qualité. Le contre-modèle est ici le «spectateur lambda » qui se contente du "tout venant » et qui sera "satisfait d'une simple pénétration » (Fabrice).

Contester la dévalorisation de la pornographie et la ridiculisation de ses publics ne conduit toutefois pas à valoriser toutes les pornographies ni à défendre tous les publics. Chez certains participants à l'enquête - tout comme parmi les journalistes et les universitaires specialisées (Paasonen, Saarenmaa, 2007) - la valorisation nostalgique d'un «âge d'or» du cinéma pornographique narratif des années 1970 passe paradoxalement par un alignement sur le discours anti-pornographie selon lequel la vidéo numérique gonzo des années 2000-2010 serait sans aucun intérêt culturel. Pierre 
(44 ans, hétérosexuel, célibataire, cuisinier, participe à un forum) trace par exemple une frontière nette entre les grands films pornographiques dont il est amateur et les vidéos pornographiques standardisées auxquelles il était «accro». Le mode de la passion est alors moins un abandon qu'un déplacement de l'échelle de valeur dominante. Se passionner pour des pornographies "esthétiques» n'est alors pas incompatible avec le rejet du « tout venant » que l'on trouve sur internet.

La passion s'actualise par ailleurs à travers des pratiques d'archivage et de partage. À l'inverse du mode de la distinction, qui vise à ne pas laisser de traces, ce mode implique la mise en scène de sa "culture " pornographique au sein d'espaces qui la valorisent. Comme dans toute enquête sur une pratique controversée et peu légitime, la relation d'enquête entre des passionnés et un sociologue devient elle-même une scène où se joue la reconnaissance de la valeur de la pratique. Pierre (44 ans, hétérosexuel, célibataire, cuisinier, participe à un forum) ponctue par exemple son explication du mode de classification des dix mille vidéos pornos qu'il compte sur son disque dur externe par l'exclamation «Ah ben attends, moi, je suis un vrai passionné! Tu crois quoi ?! ». Il rappelle ensuite son statut de membre «historique » et de "pilier» du forum de passionnés de pornographie via lequel plusieurs des participants à cette enquête ont été recrutés. Parmi les usagers de ce forum, Pierre distingue les internautes ordinaires, « des gens qui viennent pour poser des questions, pour trouver le nom d'un film », d'une élite d'internautes qualifiés :

Des gens qui ont une vraie culture, une culture extraordinaire et qui peuvent dire "C'est tel film" ou "C'est telle scène que tu cherches" et, ça, c'est exceptionnel! [...] des gens qui ont une culture pornographique énorme, qui peuvent avoir 4000 , 5000 films chez eux - je sais pas si t'imagines ce que c'est, mais c'est plus grand que le mur de ton salon [Pierre, 44 ans, hétérosexuel, célibataire, cuisinier, participe à un forum].

La connaissance de noms et la possession d'objets pornographiques rares constituent, dans l'espace restreint des sociabilités qui se nouent autour de ce forum, un capital subculturel (Thornton, 1995). Ce qui pourrait apparaître, aux yeux d'une personne non initiée, comme des objets et des images sans valeur, est requalifié au sein de l'espace du forum comme « une petite mine d'archives », pour reprendre l'expression que Bertrand (42 ans, hétérosexuel, célibataire, petits boulots, participe à un forum) utilise pour parler des divers documents qu'il a conservés au fil des années au sujet des deux actrices pornographiques auquel il consacre un blog.

La passion ne peut toutefois s'actualiser que dans les espaces où elle est reconnue à sa «juste valeur». C'est pourquoi le maintien d'un enthousiasme durable dépend de formes collectives et institutionnalisées de reconnaissance de la valeur des pratiques pornographiques. La reconnaissance par les autres membres du forum de la valeur des pratiques de Bertrand ne suffit pas à contrebalancer l'incompréhension et le dénigrement dont elles font l'objet en dehors de ce cercle restreint d'initiés. Il n'a pu partager cette passion que de manière très superficielle avec ses compagnes (il est aujourd'hui célibataire depuis sept ans). Il ne parle pas non plus de pornographie avec ses autres amis et il dissocie complètement son compte Facebook, dédié à une sociabilité culturelle généraliste, de son activité pornographique, qui se retrouve ainsi cloisonnée. Par ailleurs, les actrices pornographiques auxquelles il consacre son travail de fan montrent au mieux de l'indifférence et au pire de l'hostilité envers le travail qu'il leur consacre. Bertrand se protège en développant un rapport ironique à sa passion et en insistant sur la distance qu'il entretient à ce rôle de "fan ", un terme qu'il met à 
distance car « ça fait un peu le fanatique, avec la bave aux lèvres ». Parce que " pris au premier degré, son blog peut le faire passer pour un taré ", il atténue désormais le côté «amoureux » de son travail de passionné et en renforce le côté « ironique ». Au fil de l'entretien, l'enthousiasme et la revendication de la passion cèdent peu à peu du terrain au doute et à la tristesse. Il lui arrive en effet de ressentir de la honte à la lecture de ses écrits, dont il trouve que le côté "passionnel» est "dérangeant». Aussi il en vient parfois à penser que le culte des stars du porno ne serait qu'une "supercherie ». L'idée que la pornographie serait un monde passionnant et stimulant pour la créativité ne serait alors que « des histoires qu'on se raconte à nous-mêmes ». Cette perte de foi dans la passion pour la pornographie, que Bertrand dédramatise à travers le comique de l'absurde, l'amène à glisser progressivement du registre de la passion vers le registre du contrôle et à envisager l'énergie qu'il déploie dans les mondes de la pornographie comme un « laisser-aller».

36 Aussi fragile qu'il soit, le mode de la passion apparaît comme le plus favorable au développement d'une réflexivité critique sur ses pratiques pornographiques. La passion favorise en effet le développement d'une compréhension du travail nécessaire à la production des images, d'une familiarité avec les débats sur la pornographie et d'une écoute de la parole des actrices. Les hommes qui participent à un forum de passionnés sont en effet mieux informés de la pluralité de prises de position féministes autour de la pornographie. Aussi, faire face au stigmate $d u$ «fan » ou du «geek», qui serait un "loser " par rapport à la norme de la conquête hétérosexuelle "virile », peut être le lieu d'une prise de conscience critique sur les hiérarchies qui organisent les masculinités et, plus généralement, les rapports de genre. Bertrand (42 ans, hétérosexuel, célibataire, petits boulots) revendique par exemple une masculinité subordonnée à la puissance sexuelle des actrices, contre les injonctions à la virilité.

L'homme viril, costaud, machin, tout ça... L'image qui est vendue de l'homme... Je ne me sens pas comme ça... Je me sens resté ado [Bertrand, 42 ans, hétérosexuel, célibataire, petits boulots, participe à un forum, tient un blog].

Cette distance critique par rapport aux normes de genre est toutefois limitée par l'entre-soi masculin du forum de passionnés observé dans le cadre de cette enquête. Au début de mon entretien avec Bertrand, je précise le périmètre de mon enquête en expliquant que « je travaille sur les usages du porno par les hommes, mais [que] je fais aussi quelques entretiens avec des femmes". Bertrand me répond alors " Mais les femmes, c'est des actrices ou...? »Par réflexe et dans le tâtonnement des premiers échanges entre enquêteur et enquêté, Bertrand assimile ainsi les femmes au rôle d'actrice, c'est-à-dire, du point de vue de la réception, au rôle d'objet de séduction plutôt que sujet de désir et alter ego avec qui partager sa passion. En tant qu'administrateur de ce forum, Pierre (44 ans, hétérosexuel, célibataire, cuisinier) sait que certaines femmes s'inscrivent avec des profils masculins pour ne pas se "faire emmerder " par les « petits séducteurs qui se croiraient sur un site de rencontre ». Il se montre cependant tout aussi critique vis-à-vis des «allumeuses » qui instaurent par elles-mêmes un rapport de séduction. Aussi, si Pierre dit défendre et valoriser la participation des femmes sur ce forum de passionnés, l'engagement de celles-ci sur le territoire du langage pornographique est rapidement déploré comme un manque de féminité :

On a eu une nana qui avait vraiment un langage de charretier. Même moi, ça me... Pour moi, là, il n'y a vraiment plus de féminité... Mais ce n'est que mon point de vue. Elle parlait vraiment comme un mec... Non, moi, ça, ça me... [s'arrête] Autant 
une nana qui te dit « Vas-y bourre moi ! Salaud ! Enfile-la moi » pendant l'acte, à la limite, pourquoi pas. Ou qui aime se faire traiter de "salope ", de " grosse pute", why not. Mais arriver sur un forum avec un langage de charretier, ça me gêne. Je trouve que... Ben justement, il y a un côté féminité/masculinité qui est différent. Mais peut-être que les petites nanas qui n'ont pas d'éducation elles parlent comme des mecs hein. Mais c'est pas des gens que je fréquente. [Pierre, 44 ans, hétérosexuel, célibataire, cuisinier].

Le langage pornographique des femmes est ici accepté tant qu'il s'inscrit dans une performance de la féminité « salope », c'est-à-dire tant que les femmes restent du côté d'objet du désir (position codifiée comme féminine) et non du côté de sujet du désir (position codifiée comme masculine). Pierre apprécie ainsi la performance de la " grosse pute » ou de la « salope » dans la mesure où celle-ci s'inscrit pleinement dans l'économie genrée du désir de la pornographie hétérosexuelle conventionnelle (Damian-Gaillard, 2014). L'exclusion des femmes de cette subculture de passionnés passe par la formulation d'injonctions contradictoires : si les hommes interrogés disent valoriser la participation des femmes à ces conversations, ils insistent sur le fait qu'elles doivent s'intégrer au monde - hétérosexuel masculin - des amateurs de pornographie sans pour autant adopter une attitude masculine ou devenir l'objet du désir sexuel masculin. Cela explique sans doute qu'aucune forumeuse ne soit portée par les participants à l'enquête au panthéon des "piliers" de ce forum. Cela peut également expliquer que, selon les informations fournies par Pierre, les femmes qui assistent aux rencontres hors ligne entre "piliers » du forum (par exemple aux dîners qu'il organise) soient des actrices pornographiques et ou des compagnes de passionnés mais jamais des spectatrices à part entière. La passion pour la pornographie reste alors un plaisir d'en discuter entre hommes, sans les femmes et les féministes.

\section{Conclusion}

$\mathrm{Au}$ sein du public d'hommes de classes moyennes et supérieures étudié, les plaisirs pornographiques en ligne se vivent et se disent de quatre manières idéal-typiques. La satisfaction, en tant que valorisation circonscrite dans l'espace et dans le temps d'un plaisir simple et sans prétention, mène souvent à un refus de l'intellectualisation - et donc aussi de la politisation - de ce divertissement, vécu comme strictement intime. La distinction, c'est-à-dire la dévalorisation du plaisir pornographique en tant que vulgaire, décourage quant à elle la présentation de soi en tant que spectateur de pornographie et, ainsi, le développement d'une réflexion personnelle ou partagée sur son attachement à des formes particulières de représentation des rapports de genre. Le contrôle, en tant qu'effort pour réduire l'emprise sur son mode de vie d'un plaisir jugé dangereux, tend à reposer sur une conception purement somatique du goût et du dégoût pour la pornographie, qui défavorise l'émergence d'un questionnement politique sur l'attachement à des fantasmes de domination masculine. Enfin, si la passion favorise a priori la réflexivité sur le caractère genré de ses pratiques pornographiques, l'entre-soi masculin des sociabilités de passionnés ici observées en limite en fait fortement la portée transformative. La comparaison de ces quatre types idéaux nous amène à trois conclusions. L'analyse montre d'abord que la pornographie n'est pas illégitime en tous lieux et en tous moments; elle pose en fait surtout problème aux hommes dont le statut social dépend du capital culturel. Par ailleurs, même lorsque les hommes confèrent une valeur positive à la pornographie, leurs pratiques de 
navigation, de visionnage, de partage et de discussion restent limitées à un cercle de sociabilité restreint. Enfin, si la passion permet de s'intéresser aux enjeux féministes qui traversent la pornographie, son potentiel est bridé par l'entre soi masculin de la communauté de passionnés étudiée dans le cadre de cette enquête.

\section{BIBLIOGRAPHIE}

BAjos, Nathalie \& BOzon Michel, dir. (2008), Enquête sur la sexualité en France. Pratiques, Genre, Santé, Paris, La découverte.

BOURDIEU Pierre (1979). La Distinction. Critique sociale du jugement, Paris, Minuit.

BOzon Michel (2001), « Orientations intimes et constructions de soi. Pluralité et divergences dans les expressions de la sexualité », Sociétés contemporaines, 41-42, pp. 11-40.

BUTLER Judith (2005). Trouble dans le genre. Pour un féminisme de la subversion, trad. Cynthia Kraus, Paris, La Découverte.

CONNELL Raewyn (2014). Masculinités. Enjeux sociaux de l'hégémonie, dir. Meoïn Hagège \& Arthur Vuattoux, trad. Maxime Cervulle, Marion Duval, Clémence Garrot, Claire Richard \& Florian Vörös, Paris, Éditions Amsterdam.

CORNELL Drucila (dir). (2000). Feminism and pornography, Oxford, Oxford University Press. DAMIAN-GAILLARD Béatrice (2014). « L'économie politique du désir dans la presse pornographique hétérosexuelle masculine française ", Questions de Communication, 26, pp. 39-54.

DELEUZE Gilles (1981). Francis Bacon : Logique de la sensation, Paris, Seuil.

DYER Richard (2015). « Le porno gay, un genre filmique corporel et narratif », trad. Fred Pailler, in Florian Vörös, dir., Cultures pornographiques. Anthologie des porn studies, Paris, Éditions Amsterdam. FOUCAULT Michel (1984). Histoire de la sexualité volume 3. L'usage des plaisirs, Paris, Gallimard. GIAMI, Alain, DE COLOMBY, Patrick \& groupe ACSF (1997). « La vie sexuelle des amateurs de pornographie », Sexologies. Revue européenne de sexologie médicale, 6 (22), pp. 40-47.

GLÉVAREC Hervé (2005). « La fin du modèle classique de la légitimité culturelle ». In Éric Macé \& Éric Maigret, dir., Penser les médiacultures, Paris, Armand Colin \& INA, pp. 69-102 GOURARIER Mélanie (2017). Alpha Mâle. Séduire les femmes pour s'apprécier entre hommes, Paris, Seuil. GROSSBERG Lawrence (1992). «Is There a Fan in the House? The Affective Sensibility of Fandom » In Lisa A. Lewis, dir., The Adoring Audience : Fan Cultures and Popular Media, Londres \& New York, Routledge, pp. 50-65.

HENNION Antoine (2007). La passion musicale. Une sociologie de la médiation, Paris, Métailié. KIPNIS Laura (2015). «Comment se saisir de la pornographie? » trad. Clémence Garrot, in Florian Vörös (dir.) Cultures pornographiques. Anthologie des porn studies, Paris, Éditions Amsterdam, pp. 27-44.

LAHIRE Bernard (2004a). La culture des individus. Dissonances culturelles et distinctions de soi, Paris, La découverte. 
LAHIRE Bernard (2004b). « Individu et mélange des genres », Réseaux, 22(126), pp. 89-111.

LE BLANC ÉLIE Myriam, LAVIGNE Julie \& MAIORANO Sabrina (2017), « Cartographie des pornographies critiques ", Genre, Sexualité \& Société, $\mathrm{n}^{\circ} 17$.

MAIGRET Éric (2012). « Bande dessinée et postlégitimité » in Éric Maigret \& Matteo Stefanelli (dir.), La bande dessinée : une médiaculture, Paris, Armand Colin \& INA, pp. 130-148.

MAINA Giovanna (2013). « Grotesque Empowerment. Belladonna's Strapped Dykes entre mainstream et queer ", Rue Descartes, 79 (3), pp. 91-104.

MATHIEU Nicole-Claude (2013), « Quand céder n'est pas consentir. Des déterminants matériels et psychiques de la conscience dominée des demmes, et de quelques unes de leurs interprétations en ethnologie ", in L'anatomie politique. Catégorisations et idéologies du sexe, Paris, Ixe.

MARWICK Alice \& boyd danah (2011). « To See and Be Seen : Celebrity Practice on Twitter », Convergence. The International Journal of Research into New Media Technologies, 17 (2).

MCKEE Alan (2016). «Pornography as a creative industry: challenging the exceptionalist approach to pornography », Porn Studies, 3 (2), pp. 107-119.

MOWLABOCUS Sharif \& WOOD Helen (2015), «Introduction : audiences and consumers of porn », Porn Studies, 2(2-3), pp. 118-122.

NIXON Sean (1992). Masculinities, spectatorship \& contemporary consumption, Londres, University College of London Press.

PAASONEN Susanna \& SAARENMAA Laura (2007). « The Golden Age of Porn: Nostalgia and History in Cinema », in Susanna Paasonen, Kaarina Nikunen, and Laura Saarenmaa (dir.), Pornification. Sex and Sexuality in Media Culture, Londres, Berg. pp. 23-32.

PAASONEN Susanna, Carnal Resonance. Affect and Online Pornography, Cambridge, MIT Press, 2011.

PAILLER Fred \& VÖRÖS Florian (2017). « Des effets aux affects : médiations, pouvoir et navigation sexuelle en ligne », Revue française des sciences de l'information et de la communication, 11 [en ligne]. RUBIN Gayle (2011a). « La lutte contre la pornographie : une erreur sur toute la ligne », trad. Rostom Mesli, in Surveiller et jour. Anthropologie politique du sexe, Paris, Epel, pp. 275-315.

RUBIN Gayle (2011b), « Penser le sexe. Pour une théorie radicale de la politique de la sexualité », trad. Flora Bolter, in Surveiller et jour. Anthropologie politique du sexe, Paris, Epel, pp. 135-210

SMITH Clarissa, BARKER Martin \& ATTWOOD Feona (2015). « Les motifs de la consommation de pornographie ", trad. Lee Lebel-Canto, in Florian Vörös, dir., Cultures pornographiques. Anthologie des porn studies, Paris, Éditions Amsterdam, pp. 249-276.

SPINOZA, Baruch de (2005). Éthique, trad. Robert Misrahi, Paris, Le Livre de Poche.

TAYLOR Kris \& JACKSON Sue, «'I want that power back': Discourses of masculinity within an online pornography abstinence forum », Sexualities, 21 (4), pp. 621-636.

THORNTON Sarah (1995). « Club Cultures: Music, Media and Subcultural Capital », Cambridge, Polity Press.

TRACHMAN Mathieu (2013). Le travail pornographique. Enquête sur la production de fantasmes, Paris, La Découverte.

VÖRÖs Florian (2009). « L'invention de l'addiction à la pornographie », Sexologies, 18 (4), pp.

270-276. 
VöRös Florian (2014). « Partager l'intimité des publics. Genre, sexualité et complicité

hégémonique », Sciences de la société, 153, pp. 193-207.

WiLliams Linda (1991). «Film Bodies: Gender, Genre, and Excess », Film Quarterly, 44 (4), pp. 2-13.

Traduction française par G. Hardy et R. Nieuwjaer, disponible sur le site web Débordements. URL:

http://debordements.fr/Corps-de-film-Linda-Williams

\section{NOTES}

1. Je remercie Michel Bozon, Delphine Chedaleux, Myriam Juan, Thomas Pillard et le comité de rédaction de la revue RESET pour leurs retours précieux sur des versions successives de ce texte 2. Les déclarations de pratiques de visionnage des femmes de 25 à 49 ans décroissent à mesure que l'on monte dans la hiérarchie des catégories socio-professionnelles : le taux déclaration est d'environ $30 \%$ chez les ouvrières et les employées, d'environ $16 \%$ chez les intermédiaires dans le domaine de la santé et du travail social et les professions intellectuelles, les cadres du secteur public et les professions libérales, pour tomber à $12 \%$ chez les femmes cadres d'entreprise. De telles disparités entre les CSP ne s'observent pas dans les déclarations des hommes.

3. Narration pornographique au style brut et documentaire, souvent associé à une prise de vue subjective et des pratiques hard.

4. Pour une analyse critique des discours qui envisagent l'industrie pornographique comme une forme exceptionnelle d'exploitation économique, voir McKee (2016).

5. Pour une analyse critique des discours qui réduisent la pornographie gonzo à son sexisme, voir Maina (2013).

6. Pour une analyse critique des hiérarchies sexuelles et culturelles réactivées par le discours anti-porno, voir Rubin (2011a)

7. Ces quatre derniers entretiens font office de contrepoint au reste du corpus et l'analyse se concentre sur les pratiques des hommes cisgenres.

8. Ce biais de recrutement peut s'expliquer par le recours à des applications géolocalisées depuis le dixième arrondissement parisien, ainsi que par la diffusion des annonces de proche en proche au sein de cercles étudiants.

9. Pour une analyse réflexive de ces relations d'enquête, voir Vörös (2014). Sur les enjeux méthodologiques de l'enquête auprès des publics de la pornographie, voir Mowlabocus et Wood (2015).

10. Cette métaphore du médicament, récurrente dans mes entretiens avec des spectateurs, est également courante dans la littérature scientifique (Giami, de Colomby, et le groupe ACSF 1997).

11. Voir par exemple, Camille Emmanuelle, "Payer pour du porno: un enjeu éthique ", Les Inrockuptibles, 5 mai 2015.

12. Les Miches de la boulangère (réal. Michel Ricaud, prod. Marc Dorcel, 1993)

13. La Belle des Vosges (réal. Marc Paris et Christophe Robleur, 1997)

\section{RÉSUMÉS}

À partir d'entretiens ethnographiques conduits avec des hommes, en contextes hétérosexuel et gay, au sein de différentes fractions des classes moyennes et supérieures, cet article interroge la 
valeur culturelle accordée aux pornographies numériques. Il compare quatre modalités idéalestypiques d'organisation des plaisirs pornographiques : la satisfaction, la distinction, le contrôle et la passion. L'analyse montre d'abord que la pornographie n'est pas illégitime en tous lieux et en tous moments; elle pose en fait surtout problème aux hommes dont le statut social dépend du capital culturel. Par ailleurs, même lorsque les hommes confèrent une valeur positive à la pornographie, leurs pratiques de navigation, de visionnage, de partage et de discussion restent limitées à un cercle de sociabilité restreint. Enfin, si la passion permet de s'intéresser aux enjeux féministes qui traversent la pornographie, son potentiel est bridé par l'entre soi masculin de la communauté de passionnés étudiée dans le cadre de cette enquête.

Working from ethnographic interviews with gay, bisexual and heterosexual men, this article examines the cultural value of online pornographies. I compare four ideal-typical ways of organising pornographic pleasures: satisfaction, distinction, control and passion. The article shows that pornography is not illegitimate in all spaces and at all times; men who struggle the most with its legitimacy are actually those whose social position depends upon the public display of cultural capital. Another finding is that even viewers who do value pornography tend to limit its publicity to very specific homosocial male settings. Within the hetero porn fan community I studied, interest in feminist debates around pornography coexists with sexist attitudes towards female consumers.

\section{INDEX}

Keywords : pleasure, reflexivity, sociability, gender, masculinities, sexualities

Mots-clés : plaisir, réflexivité, sociabilité, genre, masculinités, sexualité

\section{AUTEUR \\ FLORIAN VÖRÖS}

Université de Lille 